\title{
Overview of jet quenching and energy loss in heavy-ion collisions
}

\author{
Liliana Apolinário* \\ LIP, Avenida Professor Gama Pinto, 2, 1649-003 Lisbon, Portugal \\ Instituto Superior Técnico (IST), Universidade de Lisboa, Avenida Rovisco Pais 1, 1049-001, \\ Lisbon, Portugal \\ E-mail: liliana@lip.pt
}

This talk covers the latest theory developments related to the jet-quenching phenomenon and energy loss in nucleus-nucleus collisions with a focus on the lead-lead system. In particular, an attempt is made to interpret the latest heavy-ion results in the jet physics sector using a broad range of observables e.g. inclusive $R_{A A}$, dijet asymmetry, jet fragmentation functions and sensitivity of jet substructure to jet-induced medium response.

Sixth Annual Conference on Large Hadron Collider Physics (LHCP2018)

4-9 June 2018

Bologna, Italy

${ }^{*}$ Speaker. 


\section{Introduction}

The plethora of observables based on fully reconstructed jets have demonstrated that jet quenching studies can be used as an insightful probe of the QGP medium that is created at both RHIC and the LHC. Alongside the experimental and phenomenological efforts, the theoretical description has been continuously improved. In this manuscript, it follows a short review of the latest theoretical and phenomenological developments on jet quenching.

\section{Theoretical description of jet quenching phenomena}

Although the state of matter that is created in ultra-relativistic heavy-ion collisions at RHIC and the LHC is a strongly coupled fluid, medium-induced modifications of a fast parton traversing such hot and dense medium can be understood within a perturbative approach. In this limit, the produced quark-gluon plasma (QGP) is regarded as a collection of static scattering centres with a screening potential characterised by a Debye Mass, $m_{D}$, spaced through a medium longitudinal extent $L$ such that $m_{D}^{-1} \ll \lambda_{m f p} \ll L$, being $\lambda_{m f p}$ the mean-free-path of a particle propagating through this medium. As a result of the scatterings with the medium constituents, the parton undergoes transverse momentum broadening and energy loss by medium-induced gluon radiation. Such process, initially addressed by [1,2], features most of the characteristics observed in leading particle measurements in heavy-ion collisions. However, to successfully describe jet and intra-jet observations, a theoretical treatment beyond single particle emission processes is needed.

In the last years, several works contributed towards a full in-medium parton shower picture and consequent interpretation of experimental observations. In particular, when describing a full reconstructed jet, multiple emission processes should be considered. In leading order approximation, it was shown that successive gluon emissions can be considered as independent of each other [3, 4]. In this limit, multiple emissions within the parton shower are dominated by the formation time of the emitted particles, $\tau_{\text {form }}$. In the presence of a coloured medium: $\tau_{\text {form }} \sim \sqrt{\frac{\omega}{\hat{q}}}, \theta_{b r} \sim\left(\frac{2 \hat{q}}{\omega^{3}}\right)^{1 / 4}$, where $\theta_{b r}$ is the typical emission angle, $\omega$ the energy of the radiated parton and $\hat{q}$ the transport coefficient that translates the average transverse momentum squared acquired by the particle per $\lambda_{m f p}$. The shower development is thus dominated by the fast emission of soft medium-induced gluons, that are emitted at large angles, degrading in this way the jet energy.

As single gluon emission processes seem to be the driving mechanism of the jet energy loss ${ }^{1}$, intra-jet activity can only be described if interferences between several emitters [7] are taken into account. The presence of coherent radiation sources inside of the jet depends on the scale separation between the transverse scale of the hard structure inside of the medium, $\theta_{q \bar{q}} L$, where $\theta_{q \bar{q}}$ is the opening angle of the quark-antiquark antenna, and the transverse resolution of the medium itself, $Q_{s}^{-1}=(\hat{q} L)^{1 / 2}$. When $Q_{s}^{-1}<\theta_{q \bar{q}} L$ the medium is able to probe the colour charge of both quarks and there is a complete decoherence inside of the jet, where all particles lose energy independently. In the opposite case, the effective number of emitters inside of the jet decreases (as so as medium-induced modifications to the jet structure), as only the total charge of the hard structure is probed by the medium. The interplay between these two coherent/decoherent regimes was

\footnotetext{
${ }^{1}$ See [5] for a comparison of a Monte Carlo implementation of single medium-induced gluon radiation, Q-PYTHIA [6], with dijet observables.
} 
demonstrated to be essential to capture the main features of the jet fragmentation functions [8], as measured by the LHC collaborations $[9,10]$ : high momentum particles are almost unmodified with respect to proton-proton (pp) as they are not resolved by the medium; larger transverse structures experience in-medium gluon radiation, and are therefore suppressed with respect to pp, producing soft momentum fragments.

The number of independent emitters also seems to drive the dijet asymmetry, a conclusion that holds for both perturbative QCD (pQCD) [11] and holographic [12,13] based models for in-medium energy loss. In [12], the authors considered an ensemble of light quark jets with an energy and opening angle distributions from $\mathrm{PQCD}$, that, by means of a holographic representation into a string in the dual 4+1 -dimensional gravitational theory, were introduced into hydrodynamic droplets of plasma at constant temperature. The resulting ensemble average of jet opening angle, $\left\langle C_{1}^{(1)}\right\rangle$, is shown as a function of the jet energy, $E_{j e t}$ in figure 1 . The solid black like corresponds to pp jets, followed by several model parameters in coloured dashed lines, that were tuned to provide the same jet $R_{A A}$ suppression. Like shown in [14], all jets become wider as they undergo energy loss processes. Nonetheless, the more wider is the jet (larger $a$ parameter), the larger is the shift towards small energy. Depending on the region of the energy spectrum of the initial jet ensemble, a given energy bin can be repopulated by jets that were initally more narrow, leading to an effective decrease of the $\left\langle C_{1}^{(1)}\right\rangle$ with respect to pp. These results were recently improved in [13] and allowed a comparison with jet observables, in particular the dijet asymmetry. The initial ensemble is fitted to the vacuum jet shape and jet $R_{A A}$ and, as it is possible to see from figure 2, the holographic description (solid red line) can qualitatively describe the CMS data [15] (blue points).

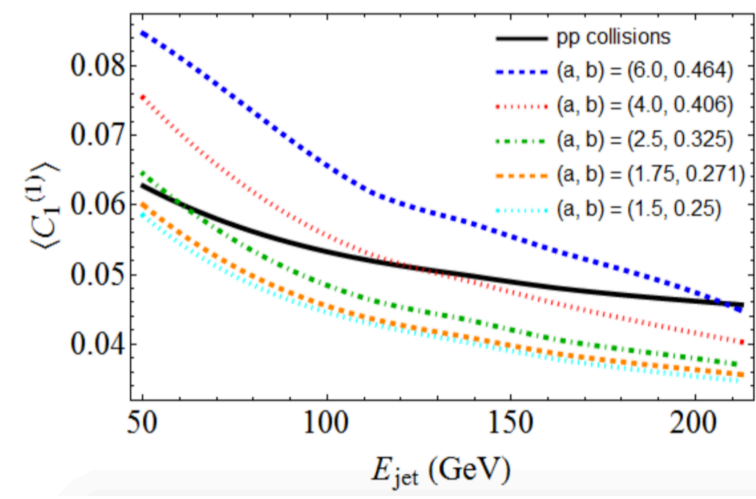

Figure 1: Ensemble average of the jet opening angle, $\left\langle C_{1}^{(1)}\right\rangle$, resulting from the propagation of an initial ensemble of jets that propagated through a strong-coupled plasma (see text for details; figure taken from [12]).

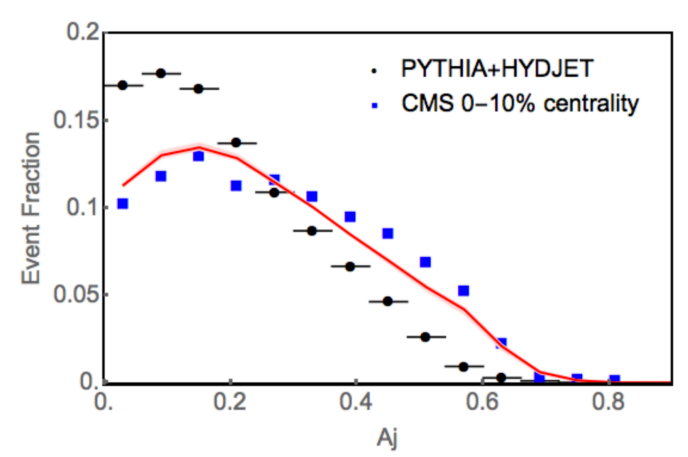

Figure 2: Resulting dijet asymmetry of an holographic ensemble of jets that propagated through a strong-coupled plasma (see text for details; figure taken from [13]).

This idea of coherent sources of energy loss inside of the jet is still not implemented in most Monte Carlo jet quenching models. Nonetheless, it is possible to have an estimate of the effect of the fluctuations of such coherent jet substructures on the final-state jet spectrum, through the jet collimator object introduced in [16]. At leading logarithmic approximation, i.e, soft and large angle emissions $\left(\theta>\theta_{c} \sim(\hat{q} L)^{-1 / 2}\right)$, it was shown that the Sudakov form factor for quark-initiated jets of 
radius $R$, can be written as $C_{q}\left(p_{T}, R\right) \simeq \exp \left[-2 \bar{\alpha} \ln \frac{R}{\theta_{c}}\left(\ln \frac{p_{T}}{\omega_{c}}+\frac{2}{3} \ln \frac{R}{\theta_{c}}\right)\right]$, when the jet transverse momentum, $p_{T}>\omega_{c}=\hat{q} L^{2} / 2$ and $C_{q}\left(p_{T}, R\right) \simeq \exp \left[-\frac{3 \bar{\alpha}}{4} \ln ^{2} \frac{R^{4 / 3} p_{T}}{\hat{q}^{1 / 3}}\right]$, for $p_{T}<\omega_{c}$ and at fixed coupling constant $\bar{\alpha}=\alpha_{s} C_{F} / \pi$. When applied to the jet suppression factor, $R_{j e t}=Q_{q}\left(p_{T}\right) \times C_{q}\left(p_{T}, R\right)$, where $Q_{q}\left(p_{T}\right)$ is the quark quenching factor in the approximation of independent soft mediuminduced radiation, the results show that even by moderating the quenching factor, substructure fluctuations induce a suppression of the jet spectrum at high- $p_{T}$. The resulting jet yield is, nonetheless, larger with respect to the independent soft medium-induced radiation.

With the furthering of our understanding on color decoherence and multiple medium-induced branchings, medium-induced radiation is under theoretical control. However, it remains a challenge to interplay this mechanism with the usual vacuum-like radiation that builds up the parton shower, from a high virtuality up to the hadronization scale. In the recent paper by [17], the effects of the medium on vacuum-like emissions are calculated in a leading double-logarithmic approximation (multiple emissions strongly ordered in angle and energy). Considering a quark-antiquark antenna setup that is propagating through a medium of size $L$, it is shown that, within such approximation, DGLAP evolution equations still apply to the description of the parton shower. The effect of the medium appears as a vetoed region on the radiation phase space (see figure 3) and, for the first emission outside the medium, angular ordering can be violated. This example is shown in figure 3 , where the last emission inside the medium is labelled "1", while the first emission outside the medium, "2", violates angular ordering. These results were used to calculate the jet fragmentation function, $D(\omega)$ (see figure 4), for different $\hat{q}$ values represented by a different color and different hadronization scale, $\Lambda$, in dashed. The main qualitative features agree with LHC measurements $[9,10]$. In particular, the suppression of intermediate energy particles with increasing $\hat{q}$ can be understood as a consequence of the vetoed region.

$\omega$

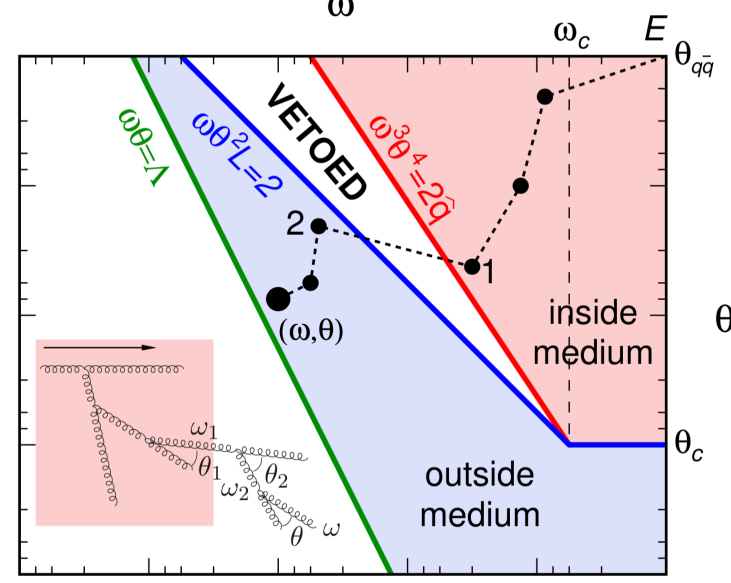

Figure 3: Schematic representation of a sequence of radiations (in dashed) in the $(\omega, \theta)$ phasespace available for vacuum-like emissions, with medium and vacuum regions identified by different colourings (see text for details, figure taken from [17]).

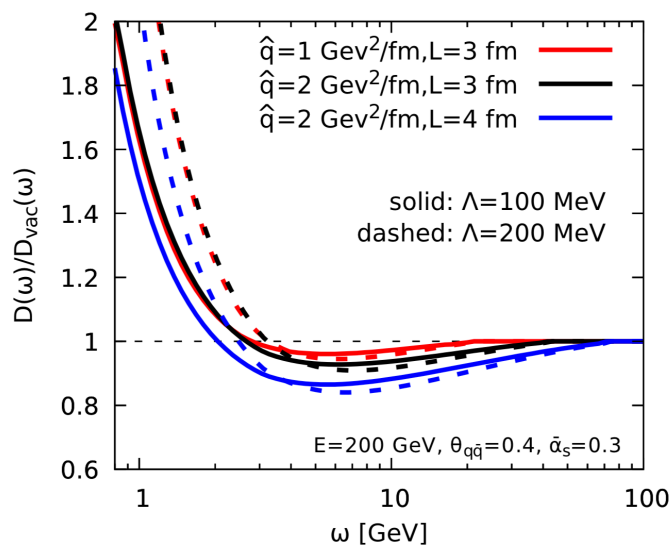

Figure 4: Ratio of the medium over vacuum jet fragmentation functions, $D(\omega) / D_{\text {vac }}(\omega)$ for different values of $\hat{q}$ and $\Lambda$ (figure taken from [17]). 


\section{Monte Carlo implementations of jet-induced medium response}

Fully reconstructed jets in heavy-ion collisions, compared with single particle measurements, have brought a unique possibility to study QCD main characteristics in a wide range of energies and densities: from the high momentum fragments that are the direct product of the partonic branches during the shower development, to the QGP part with which the jet interacts with, and that becomes correlated with the jet - known as medium response. However, there is no analytical tool in QCD that allows to have a consistent description of such multi-scale problem. In recent years, there have been several phenomenological attempts to try to evaluate the nature of such correlated background and contribution to the jet development. These have been incorporated in some Monte Carlo jet quenching models that can be categorised depending on how each model describe this jet-induced medium response: (a) As particles from a thermal or hydrodynamic particle distribution that participate in the jet-medium interaction. These recoiled particles, that in some models continue to re-scatter with the medium, become part of the jet. Models like JEWEL [18] (without medium re-scattering), MARTINI [19, 20] or LBT [21, 22] are included in this category. (b) As a source term for the (ideal/viscous) hydrodynamic evolution equation that describes the QGP development. The hydrodynamic response of the QGP due to the jet passage is afterwards converted into hadronic matter that become part of the jet. Here we find the Coupled Jet-Fluid model [23], for ideal hydrodynamics, CoLBT-Hydro [24] that considers not only viscous corrections, as it also contemplates the elastic interactions as the previous category, and to some extent, the Hybrid strong/weak coupling model [25], that instead of sourcing the energy loss, consider it instead as a perturbation in the hadronic final spectra.

There have been several works that try to identify the sensitivity of particular jet and intrajet observables due to this effect. Despite the several choices of each Monte Carlo on the chosen models and further assumptions to treat radiative and elastic energy loss processes, the introduction of a medium back-reaction always provide an increase of soft particles at distances far away from the jet core. In the listed models, this effect allow to have an overall agreement with, e.g.,missing transverse momentum ([25]), jet fragmentation functions ([25, 21, 18]), and, depending on the model characteristics, can also change the resulting jet yield ([23, 18]) and jet mass $([18,20])$. However, it is the jet shapes [26] observable that challenges any energy loss description that do not contemplate the introduction of a medium back-reaction effect. Figure 5 shows a comparison between two models from each category above: MARTINI (on the left) and Coupled Jet-Fluid (on the right). While this component is essential to describe the enhancement of particles at large angles, the evaluation of the amount of jet-induced response that is actually captured inside the jet cone is still model dependent.

\section{Summary}

There has been a significant development towards a consistent description of in-medium jet development, including parton shower modifications and thermalisation with the QGP. From current experimental results on jet and intra-jet activity, it is not yet clear what is the nature of the excess of the soft particles at large angles: jet-induced medium response or medium-induced gluon radiation from the jet development. So far, jet shapes seems to be one of the few observables that 

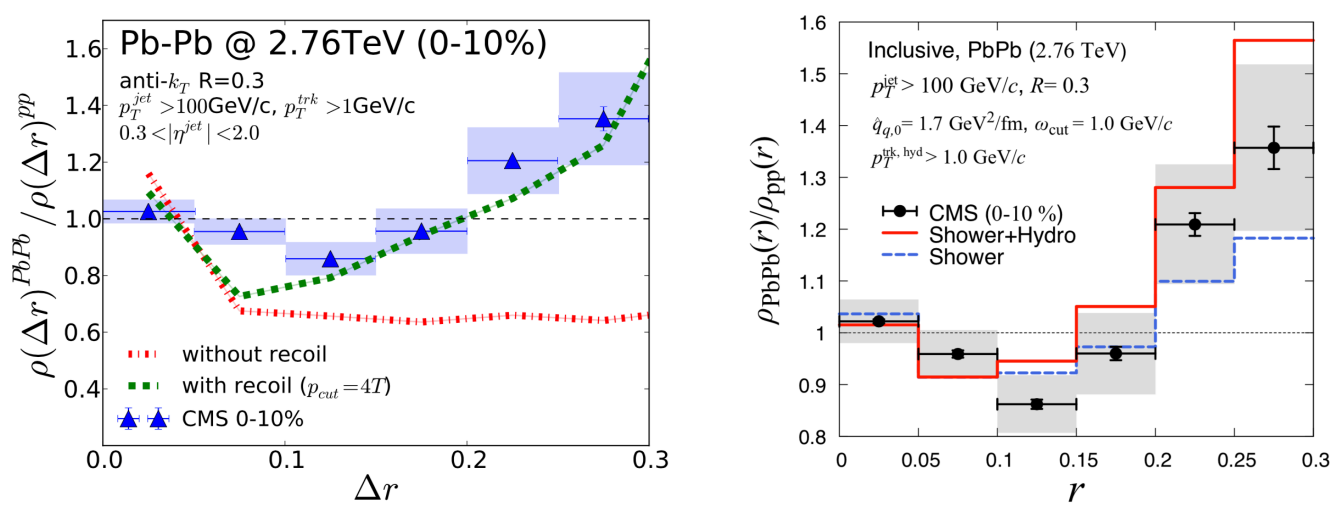

Figure 5: $\mathrm{PbPb}$ over pp ratio of the jet shape function as given by MARTINI (left) and Coupled Jet-Fluid model (right) when compared to CMS data [26]. In both models, it is shown the results with and without jet-induced medium response (figures taken from [20] and [23]).

is difficult to be explained only by parton shower modifications. A more quantitative comparison on the $p_{T}$ differential jet shapes or the use of new observables (such as $[27,28]$ ) to discriminate correlated and uncorrelated background sources might help to constrain the different models.

Acknowledgments: The author would like to thank to N. Armesto, R. Conceição, J.G Milhano, K. Tywoniuk and W. Van der Schee for insightful comments on the elaboration of this talk. This work was supported by Fundação da Ciência e Tecnologia (FCT - Portugal) under contracts SFRH/BPD/103196/2014 and CERN/FIS-NUC/0049/2015.

\section{References}

[1] R. Baier, Y. L. Dokshitzer, A. H. Mueller, S. Peigne and D. Schiff, Radiative energy loss of high-energy quarks and gluons in a finite volume quark - gluon plasma, Nucl. Phys. B483 (1997) 291 [hep-ph/9607355].

[2] B. G. Zakharov, Light cone path integral approach to the Landau-Pomeranchuk-Migdal effect, Phys. Atom. Nucl. 61 (1998) 838 [hep-ph/9807540].

[3] J.-P. Blaizot, F. Dominguez, E. Iancu and Y. Mehtar-Tani, Medium-induced gluon branching, JHEP 01 (2013) 143 [1209.4585].

[4] L. Apolinário, N. Armesto, J. G. Milhano and C. A. Salgado, Medium-induced gluon radiation and colour decoherence beyond the soft approximation, JHEP 02 (2015) 119 [14 07.0599 ].

[5] L. Apolinário, N. Armesto and L. Cunqueiro, An analysis of the influence of background subtraction and quenching on jet observables in heavy-ion collisions, JHEP 02 (2013) 022 [1211.1161].

[6] N. Armesto, L. Cunqueiro and C. A. Salgado, Q-PYTHIA: A Medium-modified implementation of final state radiation, Eur. Phys. J. C63 (2009) 679 [0907.1014].

[7] J. Casalderrey-Solana, Y. Mehtar-Tani, C. A. Salgado and K. Tywoniuk, New picture of jet quenching dictated by color coherence, Phys. Lett. B725 (2013) 357 [1210 . 7765].

[8] Y. Mehtar-Tani and K. Tywoniuk, Jet (de)coherence in PbPb collisions at the LHC, Phys. Lett. B744 (2015) 284 [1401 . 8293]. 
[9] CMS collaboration, S. Chatrchyan et al., Measurement of jet fragmentation in $\mathrm{PbPb}$ and pp collisions at $\sqrt{s_{N N}}=2.76 \mathrm{TeV}$, Phys. Rev. C90 (2014) 024908 [1 406.0932 ].

[10] ATLAS collaboration, M. Aaboud et al., Measurement of jet fragmentation in $\mathrm{Pb}+\mathrm{Pb}$ and $\mathrm{pp}$ collisions at $\sqrt{s_{N N}}=5.02 \mathrm{TeV}$ with the ATLAS detector, Phys. Rev. C98 (2018) 024908 [1805.05424].

[11] J. G. Milhano and K. C. Zapp, Origins of the di-jet asymmetry in heavy ion collisions, Eur. Phys. J. C76 (2016) 288 [1512.08107].

[12] K. Rajagopal, A. V. Sadofyev and W. van der Schee, Evolution of the jet opening angle distribution in holographic plasma, Phys. Rev. Lett. 116 (2016) 211603 [1602.04187].

[13] J. Brewer, K. Rajagopal, A. Sadofyev and W. Van Der Schee, Evolution of the Mean Jet Shape and Dijet Asymmetry Distribution of an Ensemble of Holographic Jets in Strongly Coupled Plasma, JHEP 02 (2018) 015 [1710.03237].

[14] P. M. Chesler and K. Rajagopal, On the Evolution of Jet Energy and Opening Angle in Strongly Coupled Plasma, JHEP 05 (2016) 098 [1511. 07567].

[15] CMS collaboration, S. Chatrchyan et al., Jet momentum dependence of jet quenching in $\mathrm{PbPb}$ collisions at $\sqrt{s_{N N}}=2.76$ TeV, Phys. Lett. B712 (2012) 176 [1202.5022].

[16] Y. Mehtar-Tani and K. Tywoniuk, Sudakov suppression of jets in QCD media, 1707.07361.

[17] P. Caucal, E. Iancu, A. H. Mueller and G. Soyez, Vacuum-like jet fragmentation in a dense QCD medium, Phys. Rev. Lett. 120 (2018) 232001 [1801.09703].

[18] R. Kunnawalkam Elayavalli and K. C. Zapp, Medium response in JEWEL and its impact on jet shape observables in heavy ion collisions, JHEP 07 (2017) 141 [1707. 01539].

[19] B. Schenke, C. Gale and S. Jeon, MARTINI: An Event generator for relativistic heavy-ion collisions, Phys. Rev. C80 (2009) 054913 [0909.2037].

[20] C. Park, S. Jeon and C. Gale, Jet modification with medium recoil in quark-gluon plasma, 1807.06550 .

[21] X.-N. Wang and Y. Zhu, Medium Modification of $\gamma$-jets in High-energy Heavy-ion Collisions, Phys. Rev. Lett. 111 (2013) 062301 [1302.5874].

[22] S. Cao, T. Luo, G.-Y. Qin and X.-N. Wang, Linearized Boltzmann transport model for jet propagation in the quark-gluon plasma: Heavy quark evolution, Phys. Rev. C94 (2016) 014909 [1605. 06447 ].

[23] Y. Tachibana, N.-B. Chang and G.-Y. Qin, Full jet in quark-gluon plasma with hydrodynamic medium response, Phys. Rev. C95 (2017) 044909 [1701.07951].

[24] Y. He, T. Luo, X.-N. Wang and Y. Zhu, Linear Boltzmann Transport for Jet Propagation in the Quark-Gluon Plasma: Elastic Processes and Medium Recoil, Phys. Rev. C91 (2015) 054908 [1503.03313].

[25] J. Casalderrey-Solana, D. Gulhan, G. Milhano, D. Pablos and K. Rajagopal, Angular Structure of Jet Quenching Within a Hybrid Strong/Weak Coupling Model, JHEP 03 (2017) 135 [1609. 05842 ].

[26] CMS collaboration, S. Chatrchyan et al., Modification of jet shapes in PbPb collisions at $\sqrt{s_{N N}}=2.76 \mathrm{TeV}$, Phys. Lett. B730 (2014) 243 [1310 . 0878].

[27] L. Apolinário, J. G. Milhano, M. Ploskon and X. Zhang, Novel subjet observables for jet quenching in heavy-ion collisions, Eur. Phys. J. C78 (2018) 529 [1710.07607].

[28] H. A. Andrews et al., Novel tools and observables for jet physics in heavy-ion collisions, 1808.03689. 